\title{
Study on the Factors Influencing the Preparation of Iron - Zinc Yeast Chuang Yang ${ }^{1, a}$, Zhichao Qiu ${ }^{1, b}$, Yuanwei Cao ${ }^{1, \mathrm{c}}$, Junling Wang ${ }^{{ }^{1, \mathrm{~d}}}$ \\ ${ }^{1}$ Jilin Agriculture Science and Technology University, Jilin, Jilin, 132101 \\ a yangchuang1984@163.com, ${ }^{\mathrm{b}}$ 68379293@163.com, \\ c yc2996251@163.com, d wangjunling0432@163.com
}

Keywords: Iron - Zinc Yeast; Preparation; Influencing Factors

\begin{abstract}
The enrichment of environmental elements by Saccharomyces cerevisiae was used to obtain Fe-Zn-rich yeast, and the effects of $\mathrm{pH}$, inoculation amount and culture time on Fe-Zn-Zn yeast were studied. The results showed that the influence of different factors on iron - zinc yeast culture was: culture time> $\mathrm{pH}>$ inoculation amount.
\end{abstract}

\section{Introduction}

Zinc and iron are indispensable trace elements in human and animal body, and in the body involved in a lot of important physiological and biochemical reactions. Zinc is directly involved in many important metabolic activities of organisms, such as the synthesis of protein nucleic acid, cell proliferation and differentiation, which has to promote the growth and development of the body and enhance immunity; involved in protein, fat, sugar and nucleic acid metabolism [1]; Improve taste sensitivity and improve appetite and other physiological functions. Vinson and Bose reported the relative bioavailability of supplemental inorganic salts, amino acid chelates and trace element yeasts in experimental selenium deficient, Mn deficient and zinc deficient rats. The results showed that the utilization of trace elements in yeast was the highest ]. At present, the lack of micronutrients has become the main problem of global nutritional imbalance, and yeast as the best source of trace elements supplement, increasingly favorable for people. Rich in yeast protein, its content is 2-3 times that of beef and mutton, egg 5 times, high nutritional value, and easy to absorb, rich in a variety of amino acids, including the human body 8 kinds of essential amino acids, so it is human food Ideal raw material. At present, there are many studies on the utilization of waste beer yeast, such as the use of beer yeast extract yeast extract, SOD, folic acid, fructose sodium phosphate, extracting ribonucleic acid, alkali insoluble dextran and so on. Although the natural yeast also contains a certain amount of trace elements, but can not meet the body for trace elements. Yeast cells are a good carrier of trace elements and have strong ability of adsorbing trace elements. At home and abroad, some progress has been made in the enrichment of inorganic ions in yeast, such as zinc-rich yeast, selenium-enriched yeast, [3]. The use of yeast can be trace elements from inorganic to organic, improve the biological utilization of inorganic ions, at home and abroad on yeast enrichment of single trace elements in more research, such as the development of iron-rich, rich Zinc, chromium-rich and selenium-enriched yeasts. However, the study on enriching two or more kinds of trace elements simultaneously by yeasts is still a blank. In this study, the study of yeast enrichment of trace elements iron and zinc culture conditions were explored for the future yeast enrichment of two or more trace elements to provide a theoretical basis.According to the single factor experiment, the influence of different factors on the enrichment of iron - zinc yeast was studied. The results showed that the fermented zinc - enriched yeast had the ability of enrichment of $\mathrm{Fe} 2+$ and $\mathrm{Zn} 2+$ and the influencing factors.

\section{Materials and Methods}

Beer yeast, peptone, yeast extract, glucose, potassium dihydrogen phosphate, dipotassium hydrogen phosphate, agar powder, ferrous sulfate, zinc sulfate and other microbiological laboratory provided by the Jilin Agricultural Science and Technology Institute. 
The YEPD medium was inoculated into YEPD liquid medium for 48 hours and 100 times diluted by gradient dilution method. $100 \mu \mathrm{l}$ of the bacterial solution was inoculated into YEPD solid plate medium containing $800 \mu \mathrm{g} / \mathrm{ml}$ ferrous sulfate and $200 \mu \mathrm{g} / \mathrm{ml}$ zinc sulfate, After culturing for $72 \mathrm{~h}$, a single colony with iron and zinc resistance was screened out. The strain with iron-zinc tolerance was inoculated in 500ml Erlenmeyer flask containing 50ml iron-zinc ion YEPD liquid medium. The cell was incubated at $180{ }^{\circ} \mathrm{C}$ for 28 hours at $28{ }^{\circ} \mathrm{C}$ for $12 \mathrm{~h}$. After centrifugation at 6000rpm for 20min, Water washing to no iron and zinc ions, drying to constant weight, weighing 0.2 to $0.3 \mathrm{~g}$ after the remaining frozen reserve. The contents of iron and zinc in yeast cells were determined by atomic absorption spectrometry, and the strains with high content were selected as the tested strains.

Saccharomyces cerevisiae were inoculated in $500 \mathrm{ml}$ Erlenmeyer flasks containing $50 \mathrm{ml}$ of culture medium at $800 \mu \mathrm{g} / \mathrm{ml}$ iron ion concentration and $400 \mu \mathrm{g} / \mathrm{ml}$ zinc ion concentration. Cultured with shaking at $180 \mathrm{rpm}$ for 3, 6, 8, 12, 18, 24, 36, 48, 60 and 72 hours, respectively. The fermentation broth was centrifuged at 6000rpm for $20 \mathrm{~min}$, washed with distilled water, centrifuged and repeated 3 times. After $60{ }^{\circ} \mathrm{C}$ constant temperature drying, the biomass of yeast was measured.

The initial $\mathrm{pH}$ of the fermentation medium was adjusted to 4.0, 4.5, 5.0, 5.5, 6.0, 6.5, 7.0 and 7.5 respectively. The volume of the $500 \mathrm{ml}$ triangular bottle was $50 \mathrm{ml}$, the inoculation amount was $2 \%$, the iron ion concentration was $800 \mathrm{~g} / \mathrm{Ion}$ concentration of $400 \mu \mathrm{g} / \mathrm{ml}$, 180rpm shaking culture 36h. The fermentation broth was centrifuged at 6000rpm for $20 \mathrm{~min}$, and then centrifuged three times with distilled water. After $60{ }^{\circ} \mathrm{C}$ constant temperature drying, the biomass of yeast was measured.

In a $500 \mathrm{ml}$ Erlenmeyer flask, $50 \mathrm{ml}$ of a fermentative medium was charged with an iron ion concentration of $800 \mathrm{~g} / \mathrm{ml}$, a zinc ion concentration of $400 \mathrm{~g} / \mathrm{ml}$, and a $\mathrm{pH}$ of 5.5. The strain was inoculated into the above medium at the volume ratio of $2 \%, 4 \%, 6 \%, 8 \%$ and $10 \%$, and cultured at $180 \mathrm{rpm}$ for $36 \mathrm{~h}$. The fermentation broth was centrifuged at $6000 \mathrm{rpm}$ for $20 \mathrm{~min}$, and then centrifuged three times with distilled water. After $60{ }^{\circ} \mathrm{C}$ constant temperature drying, the biomass of yeast was measured.

\section{Results and Analysis}

According to Figure 1, we can see: yeast in 0 to $4 \mathrm{~h}$ in the induction period of the number of cell growth was not obvious 5-36h in the logarithmic growth phase, cell growth speed, strong growth ability to adapt to strong, after $36 \mathrm{~h}$ into a stable period, After $60 \mathrm{~h}$, the cell concentration was basically constant. After 72 hours, the nutrient condition decreased, the yeast died and died, and the number of cells decreased.

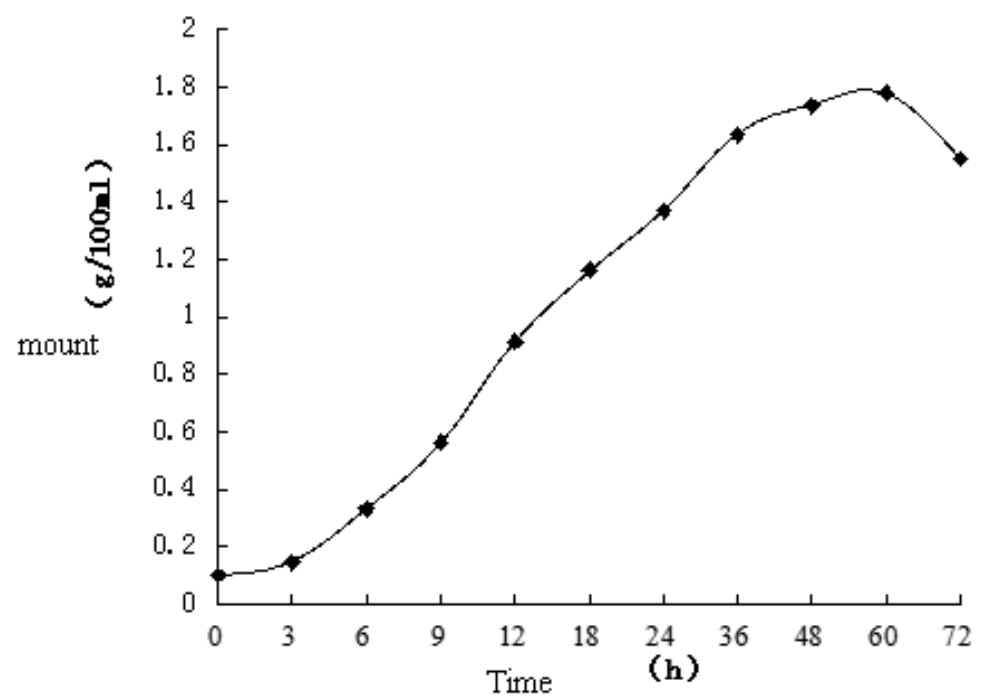

Fig.1 Effect of incubation time on yeast biomass

Figure 2 shows that with the increase of $\mathrm{pH}$, the growth rate of yeast increased and biomass increased continuously. Under different $\mathrm{pH}$ conditions, the growth curve of yeast was ridiculous, and the most vigorous acidity was between 5.0 and 6.5. . Brady and Duncan [4], Fourest and Roux 
[5] studies have shown that the optimum $\mathrm{pH}$ range for microbial uptake of metal ions is between 4.0 and 8.0.

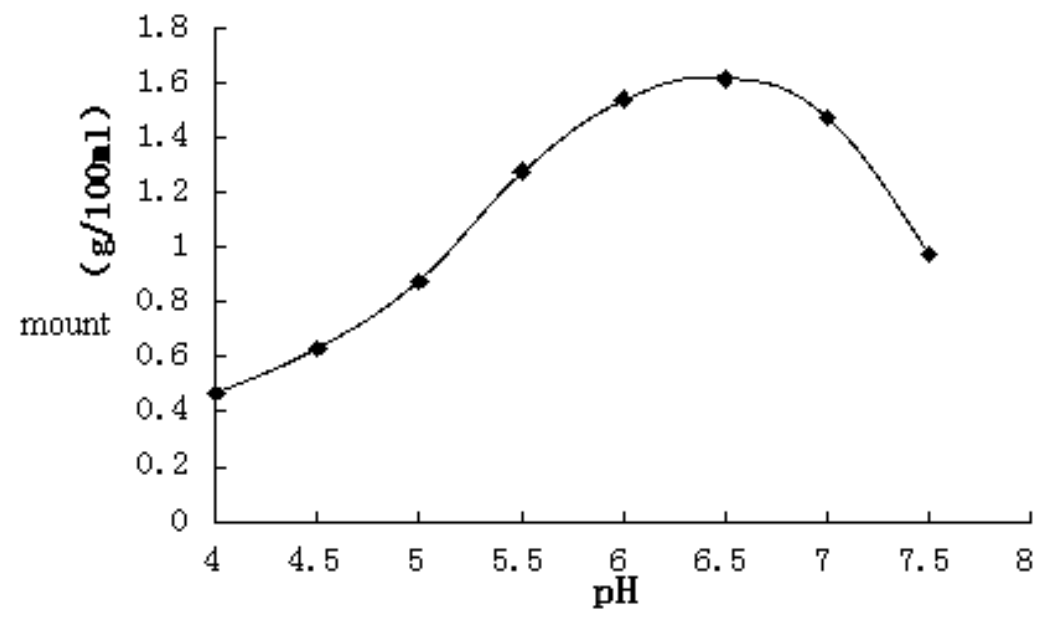

Fig. 2 Effect of $\mathrm{pH}$ on yeast biomass

From Fig. 3, the inoculum size had a great influence on the biomass accumulation and metal ion enrichment of S. cerevisiae, and the biomass reached the maximum at 6\%. Inoculation amount is too large can inhibit the biomass and iron and zinc ion enrichment.

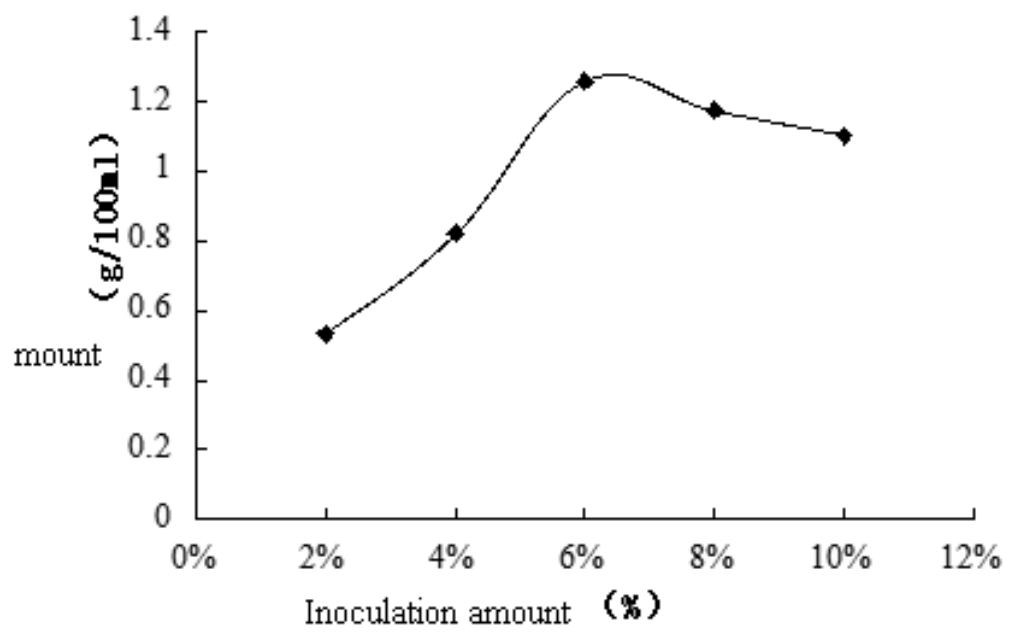

Fig. 3 Effect of inoculum size on yeast biomass

According to the single factor experiment, the influence of ferric - zinc yeast culture time was: culture time $>\mathrm{pH}>$ inoculation amount.

Under the optimal conditions, the fermentation was carried out in a 3L Erlenmeyer flas $\mathrm{k}$, and the biomass was measured every 2 hours. The iron-zinc-yeast growth curve is plotte d (Figure 4). At $36 \mathrm{~h}$, the culture reached the stationary stage. The biomass was $16.94 \mathrm{mg} /$ $\mathrm{ml}$, the zinc content and conversion rate were $5.70 \mathrm{mg} / \mathrm{g}$, the content of iron and the conve rsion rate were $16.82 \mathrm{mg} / \mathrm{g}$. Masaru studies have shown that the appropriate concentration o $\mathrm{f}$ iron and zinc ions concentration can increase the yield of yeast [6].

There are many factors that affect the enrichment of trace elements in yeast. Due to th e limitation of experimental time and experimental conditions, the present study did not carr $\mathrm{y}$ out a comprehensive experiment on the basis of previous studies, so the conclusions coul $\mathrm{d}$ be Is not the best conclusion. The enrichment of $\mathrm{Fe}$ and $\mathrm{Zn}$ by Saccharomyces cerevisiae has provided some basis for the enrichment of micro-elements in yeast. The selection of b acteria and the optimization of culture conditions need to be further studied. Yeast enrichme nt of micro-elements and the enrichment of micro-or micro-elements of yeast in food applic ations will be the next hot spot, which has broad prospects. 


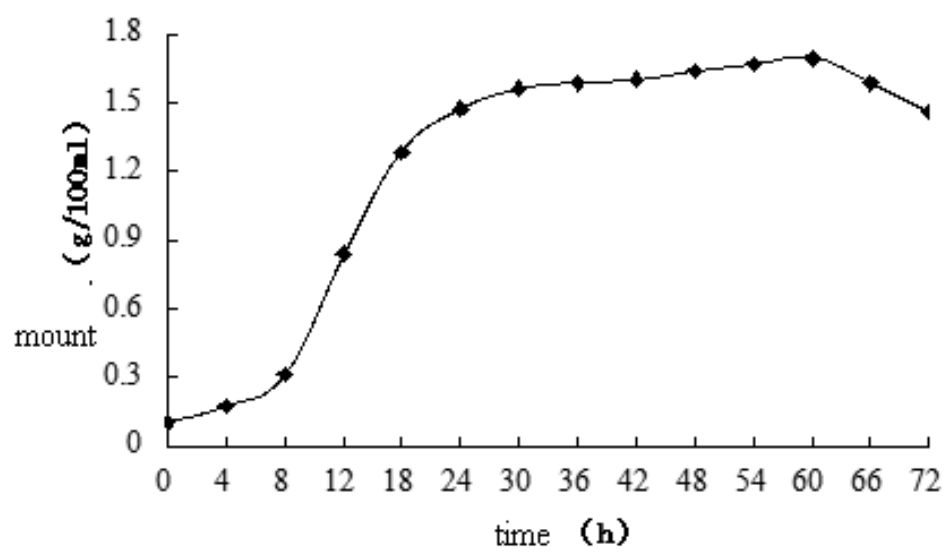

Fig. 4 Growth curve of rich Fe - Zn - yeast

\section{Acknowledgements}

Jilin Agricultural Science and Technology Institute of key disciplines to cultivate the project JNHYZ [2015] No. X066

Jilin Province College Students Innovation and Entrepreneurship Training Program Project JNYHZ [2016] No. 092

\section{References}

[1] Symons M C R, Guteridge J M C. Free Radicals and Iron: Chemistry, Biology, and Medicine [M].Oxford: Oxford Science Publications, 1998.

[2] Vinson, J. A. and Bose, Comparision of the bioavaility of trace element in Inorganic salts, Amino acid chelates and Yeast [J]. P. Proceedings on Mineral Elements, 1981, 615- 621.

[3] S. N. Dodic, S. D. Popov and S. l. Markov Investigation of kinetics of zinc biosorption by Saccharomyces cerevisiae cells [J]. Nahrung 2001, 45(1): 59- 61.

[4] Brady D, Duncan J R. Bioaccumulation of metal cations by Saccharomyes cerevisiae [J]. Appl Microbiol Biotechnol, 1994, 41(1):25 27.

[5] Fourest E J. Microbial transport of toxic metals [C]. Wiley-Liss. Environ Microbiol. New York,1992:83 101

[6]Masaru, Higasa, Shizu. Zinc and iron bio-availability using zinc/iron-enriched bakers' yeast[J]. Joshi Eiy o DaigakuKiy o, 1999, 30:159 165. 